\title{
Effect of Resveratrol as Caloric Restriction Mimetic and Environmental Enrichment on Neurobehavioural Responses in Young Healthy Mice
}

\author{
Mustapha Shehu Muhammad, ${ }_{1}^{1}$ Rabiu Abdussalam Magaji, ${ }^{2}$ Aliyu Mohammed, \\ Ahmed-Sherif Isa, ${ }^{2}$ and Mohammed Garba Magaji ${ }^{3}$ \\ ${ }^{1}$ Department of Human Physiology, College of Medical Sciences, Gombe State University, Gombe 760214, Nigeria \\ ${ }^{2}$ Department of Human Physiology, Faculty of Medicine, Ahmadu Bello University, Zaria 810001, Nigeria \\ ${ }^{3}$ Department of Pharmacology and Therapeutics, Faculty of Pharmaceutical Sciences, Ahmadu Bello University, Zaria 810001, Nigeria
}

Correspondence should be addressed to Mustapha Shehu Muhammad; msmuhammad@gsu.edu.ng

Received 17 May 2014; Accepted 6 August 2014; Published 18 August 2014

Academic Editor: Valerio Magnaghi

Copyright (C) 2014 Mustapha Shehu Muhammad et al. This is an open access article distributed under the Creative Commons Attribution License, which permits unrestricted use, distribution, and reproduction in any medium, provided the original work is properly cited.

\begin{abstract}
Caloric restriction and environmental enrichment have been separately reported to possess health benefits such as improvement in motor and cognitive functions. Resveratrol, a natural polyphenolic compound, has been reported to be caloric restriction mimetic. This study therefore aims to investigate the potential benefit of the combination of resveratrol as CR and EE on learning and memory, motor coordination, and motor endurance in young healthy mice. Fifty mice of both sexes were randomly divided into five groups of 10 animals each: group I animals received carboxymethylcellulose (CMC) orally per kg/day (control), group II animals were maintained on every other day feeding, group III animals received resveratrol $50 \mathrm{mg} / \mathrm{kg}$, suspended in $10 \mathrm{~g} / \mathrm{L}$ of (CMC) orally per kg/day, group IV animals received CMC and were kept in an enriched environment, and group V animals received resveratrol $50 \mathrm{mg} / \mathrm{kg}$ and were kept in EE. The treatment lasted for four weeks. On days 26, 27 , and 28 of the study period, the animals were subjected to neurobehavioural evaluation. The results obtained showed that there was no significant change $(P>0.05)$ in neurobehavioural responses in all the groups when compared to the control which indicates that $50 \mathrm{mg} / \mathrm{kg}$ of resveratrol administration and EE have no significant effects on neurobehavioural responses in young healthy mice over a period of four weeks.
\end{abstract}

\section{Introduction}

Dietary restriction (DR), otherwise known as caloric restriction (CR), has been generally defined as consumption of nutritious diet that is $30 \%$ to $40 \%$ less in calories compared to ad libitum diet [1]. In other words, CR can be defined as a simple reduction in caloric intake in the absence of malnutrition [2]. Caloric restriction has been demonstrated to possess many health benefits. It provides protection against numerous deadly diseases such as cancer, neurological disorders, and obesity and is found to be the only reliable treatment that extends lifespan or causes healthy aging consistently in a multitude of organisms ranging from bacteria to monkeys [3-5].
The most frequently mentioned effect of CR has been its influence on creating a mild stress in the organism and a typical upregulation of adaptive mechanisms involving stress proteins accompanied by elevated defence or survival molecules [6]. Caloric restriction (CR) has also been found to retard several aspects of the aging process in mammals, including age-related mortality, tumorigenesis, physiological decline [7], and the establishment of age-related transcriptional profiles [8].

Resveratrol (3,5',4-trihydroxystilbene), a natural polyphenolic compound found mainly in the skin of grapes and red wine, has previously been shown to extend lifespan in yeast (Saccharomyces cerevisiae), nematode worm (Caenorhabditis elegans), fruit fly (Drosophila melanogaster), 
and short-lived fish (Nothobranchius furzeri) through a sirtuins 1 dependent mechanism [9-13]. Studies have shown that mice fed with a high fat diet supplemented with high levels of resveratrol were shown to have extended lifespan compared to the control animals and several metabolic alterations similar to what is observed with CR; thus, resveratrol is said to have caloric restriction-like properties $[2,14]$.

Environmental enrichment (EE) is defined as a sustained and progressive increase in cognitive and sensorimotor stimuli with aggregated voluntary physical activity and complex social interactions [15]. Abundant experimental evidence shows that EE is beneficial in various animal models of neurodegenerative disorders such as stroke and Alzheimer's disease [16, 17]. Environmental enrichment also induces neuroprotection against 1-methyl-4-phenyl-1,2,3,6tetrahydropyridine (MPTP) toxicity to mice $[18,19]$ and improves motor function after unilateral 6-OHDA injection in rats [20]. Numerous data suggest that synthesis and release of trophic factors (TFs) may play a crucial role in mediating the neuroprotective effect of EE [16]. Environmental enrichment alters the expression of TFs and their receptors in several brain areas [21, 22] and induces astrogliogenesis [23]. In the nigrostriatal system, EE-housed animals show increased brain-derived neurotrophic factor (BDNF) expression in the striatum [18] and glia cell-line derived neurotrophic factor (GDNF) mRNA in the substantia nigra (SN) [19].

Studies have shown that $\mathrm{CR}$ and its mimetics such as resveratrol improve motor coordination and increase longevity in mice which may be related to the improvement in endurance and strength $[2,14,24]$. Resveratrol has also been shown to prevent cognitive decline in a number of disease models and to reduce neurodegeneration in vivo [25]. However, numerous works have been done on EE, $\mathrm{CR}$, and its mimetics such as resveratrol and sulforaphane $[1,24]$, and much attention has been lavished on the effect of resveratrol, $\mathrm{CR}$, and $\mathrm{EE}$ on aging and age related disorders but the possible beneficial role in young healthy animals has not been investigated. The beneficial role of CR in the nervous system has been extensively studied in aged animals [26]. Moreover, several reports showed the physiological, neurological, and behavioural effects of CR and EE in rodents, but the majority of efforts have been focused on the beneficial effects of $\mathrm{CR}$ and $\mathrm{EE}$ on aging and lifespan in the past decades, and little attention has been paid to the physiological effects of CR and EE especially in young healthy animals. Furthermore, there are controversial reports on the effects of $\mathrm{CR}$ and $\mathrm{EE}$ on cognition in young animals. Some investigators demonstrated that CR enhances learning and memory [27], while others have reported that CR had negative effects on cognitive functions [28]. Environmental enrichment has also been reported to improve spatial memory in aged mice with no significant effect in young and middle-aged mice [29]. Collectively, the available data suggest that both CR and EE exert similar beneficial effects on neurons in the brain and share a mechanism involving increased neurotrophic factor production [30]. Hence this study evaluates the effects of resveratrol as CR mimetic and EE on motor coordination, motor endurance, memory, and learning in young healthy Wistar albino mice over a period of four weeks.

\section{Materials and Methods}

2.1. Chemicals/Reagents. Resveratrol (60 g) of analytical grade was purchased from Candlewood Stars Incorporated, Danbury, USA (batch number: MR120718). Carboxymethylcellulose CMC (10 g) (product number: 27929, BDH Chemicals Ltd., Poole, UK) was obtained from Department of Pharmacology and Therapeutics, Ahmadu Bello University, Zaria. trans-Resveratrol, due to its low solubility in water, was suspended in $10 \mathrm{~g} / \mathrm{L} \mathrm{CMC} \mathrm{[31].}$

2.2. Animals. Fifty (50) Wistar albino mice of both sexes, 4 weeks of age and weighing 15-22 g, were used for this study. The animals were obtained from the Institute of Veterinary Research, Vom, Jos Plateau State. The animals were housed in standard polypropylene cages in groups of five in a temperature and humidity controlled environment subject to a $12 \mathrm{~h}$ light/dark cycle and fed with standard laboratory animal feed and water ad libitum. Dietary delivery groups were given controlled access to food; water was available ad libitum. The mice were allowed to acclimatize to the environment of the behavioural laboratory for the period of one week before commencement of the experiment. All experimental protocols were in accordance with the Ahmadu Bello University research policy and ethic and regulations governing the care and use of experimental animals (NIH publication number 85-23, revised 1996). The experiments were conducted in a quiet laboratory between hours of $900 \mathrm{~h}$ and $1600 \mathrm{~h}$.

2.3. Animal Housing and Management. The enriched cage $(66 \mathrm{~cm}$ long $\times 46 \mathrm{~cm}$ wide $\times 38 \mathrm{~cm}$ high $)$ as described by Harburger et al. [29] was used in the study. The cage contained tubes, ramps, stairs, and different "toys" (hard plastic balls, cubes, cones, and sticks). The toys were changed twice a week to continuously encourage exploration of the environment. The complexity (number of objects) of the housing facility was increased progressively: every 2 days, two to four objects were added to the environment. Ten days after housing animals in the EE, the complexity of the cage is expected to be maximal, but the positions of the objects were changed continuously after every 2 days [15]. Five (5) mice were housed together to allow social interactions. The impoverished condition consisted of normal cages $(45 \mathrm{~cm}$ long $\times 30 \mathrm{~cm}$ wide $\times 18 \mathrm{~cm}$ high) made without objects or running wheels, housing five (5) animals per cage.

Animals were kept in EE housing for four weeks while receiving the appropriate treatment, as described by Steiner et al. [23]. The control animals were given carboxymethylcellulose (CMC) and kept under good housing condition. Neurobehavioural study was carried out for the period of three (3) days during the last phase of the experiment.

2.3.1. Animal Groupings. The animals were divided into five (5) groups each comprising ten (10) animals of both sexes per group, that is, five (5) males and five (5) females mice kept in separate cages to prevent mating. Group I animals received carboxymethylcellulose (control group) $50 \mathrm{mg} / \mathrm{kg}$ per body weight, group II animals were maintained on every 
other day feeding (EODF) with standard laboratory animals diet, group III animals received resveratrol $50 \mathrm{mg} / \mathrm{kg}$ orally for four weeks as described by Blanchet et al. [32], group IV animals received CMC and were kept in an enriched housing (EE) for four weeks, and group $\mathrm{V}$ animals received resveratrol $50 \mathrm{mg} / \mathrm{kg}$ orally and were kept in EE housing (for 4 weeks).

2.4. Neurobehavioural Assessments. The following neurobehavioural studies were carried out for three days on days 26 , 27 , and 28 of the experimental period.

2.4.1. Beam Walk Test. The beam walk test was used to test motor coordination. Briefly, the beam walk apparatus consists of a beam, ruler, goal box, and an elevated wooden stand. The beam is made of wood, $8 \mathrm{~mm}$ in diameter, $80 \mathrm{~cm}$ long, and elevated $30 \mathrm{~cm}$ above the bench by a wooden support. Mice were allowed to walk from a start platform along a ruler $(80 \mathrm{~cm}$ long and $3 \mathrm{~cm}$ wide) elevated $30 \mathrm{~cm}$ above the bench by a wooden support to the goal box (enclosed hamster house). Several trials were performed for each mouse and it was designed such that the mice tested were aware that there is a goal box that should be reached. A ruler was used to train the mice and once the mice find it easy to cross, they were moved immediately to the beam [33]. The mice were placed on the beam at one end and allowed to walk to the goal box. Mice that fall from the beam were returned to the position they fell from with a maximum time of 60 seconds allowed on the beam. The measurements that were taken were time on the beam, the number of foot slips (one or both hind limbs slipped from the beam), and the number of falls. After each trial, the maze was wiped with a cotton wool dipped in $70 \%$ ethyl alcohol and allowed to dry to remove any olfactory clue or odour.

2.4.2. Hang Test. The hang test was used to assess muscular endurance in mice as described by Mohanasundari et al. [34]. Briefly, the apparatus consists of a horizontal grid (grid $12 \mathrm{~cm}^{2}$, opening $0.5 \mathrm{~cm}^{2}$ ). The grid was mounted $20 \mathrm{~cm}$ above a hard surface, to discourage falling or injury in case of falling. The apparatus was equipped with a 3-inch wall to prevent animals from traversing to the upper side of the grid. The mice were placed on the horizontal grid and supported until they held the grid. The grid was then inverted so that the mice were allowed to hang upside down. The mice were allowed to stay on the grid for $30 \mathrm{~s}$ and 10 chances were given with $1 \mathrm{~min}$ interval and the best maximum hanging time was recorded. The percentage of success was recorded as maximum time hanging/30 $\mathrm{s} \times 100$ [35].

2.4.3. Elevated Plus Maze. The elevated plus maze was used to evaluate short-term spatial memory [36]. Briefly, the elevated plus maze for mice consists of two perpendicular open arms $(21.5 \times 7.5 \mathrm{~cm})$ and two closed arms $(21.5 \times 7.5 \times 20 \mathrm{~cm})$ which extends from a central $7.5 \times 7.5 \mathrm{~cm}$ platform. The platform and floor were made from wood, and the lateral walls of the closed arms were made of wood painted black. The maze was elevated $38 \mathrm{~cm}$ above the floor. On the first day (training), each animal was placed at the end of one
TABLE 1: Effect of resveratrol as CR mimetic and EE on motor coordination of mice.

\begin{tabular}{lcc}
\hline Groups & Number of foot slips & $\begin{array}{c}\text { Time taken to complete } \\
\text { task (seconds) }\end{array}$ \\
\hline Control (CMC) & $1.13 \pm 0.44$ & $9.63 \pm 1.32$ \\
EODF & $1.30 \pm 0.42$ & $9.10 \pm 1.39$ \\
Resveratrol only & $1.20 \pm 0.42$ & $8.80 \pm 1.46$ \\
EE only & $0.88 \pm 0.44$ & $8.13 \pm 1.30$ \\
Resveratrol + EE & $0.30 \pm 0.21$ & $8.40 \pm 1.94$ \\
\hline
\end{tabular}

Results were presented as mean \pm SEM; CMC: carboxymethylcellulose; EODF: every other day feeding; EE: environmental enrichment; $n=10$.

open arm, facing away from the central platform. The transfer latency of the mouse to move from the open to the enclosed arms was recorded within $90 \mathrm{~s}$. Following entry into the arm, the animals were allowed to explore the apparatus for $30 \mathrm{~s}$. Twenty-four hours later, the second trial (retention test) was performed and the animals were observed for $90 \mathrm{~s}$. After each trial, the maze was wiped with a cotton wool dipped in $70 \%$ ethyl alcohol and allowed to dry to remove any olfactory clue or odour.

2.5. Statistical Analysis. Data obtained were expressed as mean \pm SEM. Statistical analysis was carried out using SPSS version 17 and all the analysis was done using one way ANOVA followed by Tukey's post hoc test for multiple comparisons. Values of $P<0.05$ were considered significant.

\section{Results}

3.1. Beam Walk Test. Table 1 represents the results observed in the beam walk test. A slight decrease in the number of foot slips was observed in the EE and RESV + EE groups, when compared to the control, though no statistical significance was recorded. On the other hand, an increase in number of foot slips was observed in EODF group when compared to the control; this also was not statistically significant (Table 1). There was no significant change in transfer latency on the beam among the groups, but lower values were recorded for the RESV, EE, and RESV + EE groups, respectively, when compared to the control group.

3.2. Hang Test. Figure 1 shows the changes in motor strength in the hang test. The percentage of hanging time was slightly lower in all the groups than the control, although it was not statistically significant.

3.3. Elevated Plus Maze. Table 2 shows the results obtained on the effect of resveratrol induced CR and EE on memory of mice. Result obtained showed no significant statistical difference in the various treatment groups when compared to the control, although there was slight decrease in transfer latency (retention) in the EODF, RESV, EE, and RESV + EE groups compared to the control group. 


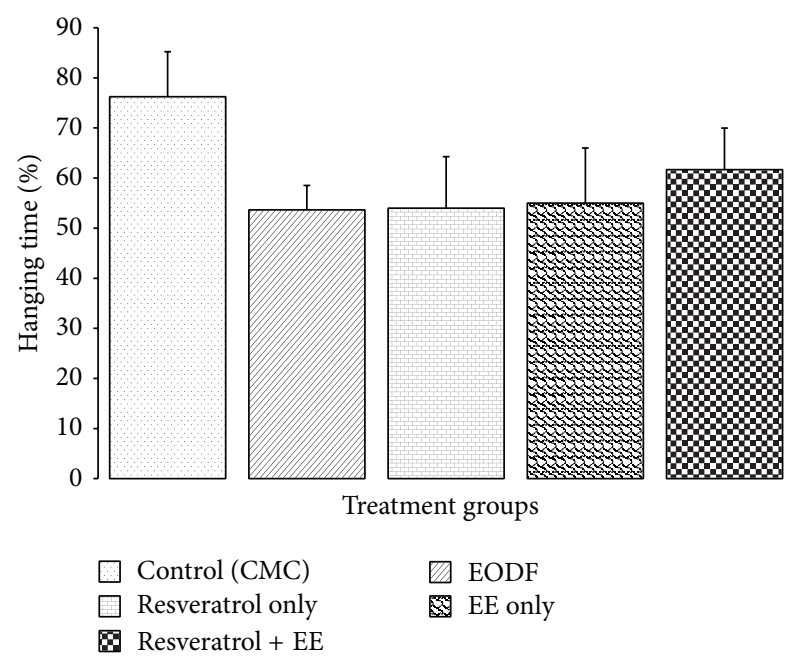

FIGURE 1: Effect of resveratrol as CR mimetic and EE on muscular endurance of mice. Results presented as mean \pm SEM; $n=10$.

TABLE 2: Effect of resveratrol as CR mimetic and EE on memory of mice.

\begin{tabular}{lcc}
\hline Groups & $\begin{array}{c}\text { Transfer latency } \\
\text { (acquisition) }\end{array}$ & $\begin{array}{c}\text { Transfer latency } \\
\text { (retention) }\end{array}$ \\
\hline Control (CMC) & $39.75 \pm 6.62$ & $23.38 \pm 9.64$ \\
EODF & $53.70 \pm 7.56$ & $17.20 \pm 4.18$ \\
Resveratrol only & $44.60 \pm 9.57$ & $14.80 \pm 3.04$ \\
EE only & $25.25 \pm 6.43$ & $10.25 \pm 1.32$ \\
Resveratrol + EE & $25.70 \pm 7.90$ & $20.40 \pm 8.01$ \\
\hline
\end{tabular}

Results were presented as mean \pm SEM; CMC: carboxymethylcellulose; EODF: every other day feeding; EE: environmental enrichment; $n=10$.

\section{Discussion}

The results obtained from this study demonstrated a trend towards improvement in motor coordination in the environmental enrichment and resveratrol treated group in an enriched environment using beam walk test, although the decrease in number of foot slips and latency were not statistically different when compared to the control group. The results of muscular endurance using hang test were also not significantly different between the groups. This is contrary to the findings of Anandhan et al. [37] who reported that resveratrol improves motor endurance using hang test in MPTP challenged mice. Both resveratrol and environmental enrichment have also been reported to extend lifespan and improve motor function in drosophila and rat models of Parkinson's disease, respectively, by activating sirtuins gene $[15,38]$.

Caloric restriction and $\mathrm{EE}$ have been found to attenuate age-related deficits in learning and memory $[15,39]$ and increase resistance of neurons to excitotoxic, oxidative, and metabolic insults and improve behavioural outcomes in experimental models of Alzheimer's and Huntington's disease [40]. The result obtained from this study using the elevated plus maze for memory, though not statistically significant, showed a slight decrease $(P>0.05)$ in the transfer latency (retention) across the groups when compared to the control with the least mean value obtained in the EE group. This is in agreement with the findings of Harburger et al. [29] who found no significant change in spatial memory of young male mice when compared to the control after exposing them to enriched environment for a period of one month. This difference was, however, attributed to the age as well as the duration of exposure of the animals to enriched environment [29].

Although, in this study, resveratrol was used as a caloric restriction mimetic to stimulate caloric restriction, the results obtained on the effect of resveratrol as CR mimetic on memory of mice were in corroboration with the findings of Deng et al. [26] who reported no significant change in short-term memory of mice using the passive avoidance test for memory after subjecting mice to a $20 \%$ caloric restriction for one month. However, no uniform conclusion has been reached on the effect of caloric restriction or caloric restriction-like effects on cognitive functions in young animals. Nevertheless, some investigators have suggested that caloric restriction enhanced learning and memory in young animals [27, 41, 42], while others reported the contrary [28, 43]. This discrepancy was attributed to several factors including differences in caloric restriction treatment, duration of caloric restriction, species or strain of animal, and type of behavioural test [26].

There is little evidence that resveratrol enhances cognition in healthy individuals in the absence of pathological or age-related cognitive decline which indicates that resveratrol confers some protection on the brain from the effects of aging and oxidative, physical, and chemical damage to the brain without measurably improving cognition in the short term [44]; this could possibly be an explanation for the absence of an observed effect of resveratrol as CR mimetic in our study. If resveratrol can only protect the brain against damage (as opposed to being able to enhance its function), it would not be expected to produce a measurable effect on cognition in young healthy animals, such as the mice in our study, and one logical explanation for the results of these studies is that resveratrol prevented the neurological pathology that is known to increase with age [45-48] but did not enhance cognition when the animals were young. The sensitivity of the neurobehavioural test used to assess memory in young healthy animals in our study could also be considered as a factor that hinders any feasible change in memory between the treatment groups and the control.

Another possible explanation for the lack of an observed effect of resveratrol as $\mathrm{CR}$ mimetic in our study was the short duration of treatment. The mice received resveratrol for only four weeks before testing began, while in previous studies, where resveratrol was shown to improve cognitive functions, the subjects were given resveratrol for at least onethird of their lifespan [13, 49]. Short periods of resveratrol treatment in rats have been shown to produce positive effects on cognition, but the animals used in those studies had been subjected to neurological trauma or treatment with a neurotoxic substance $[50,51]$. The failure to observe an effect after short-term treatment is a logical outcome if resveratrol's effect on cognitive function is mediated through 
protecting the brain against damage [44]. In previous studies where cognitive function was assessed in healthy animals, a significant effect of resveratrol was only observed in middleaged or aged animals $[13,49]$.

The failure to observe an effect of resveratrol as CR mimetic may also have been due to the combined effects of both EE and resveratrol in our procedures. Results obtained in resveratrol and EE treated groups showed decrease in transfer latency though not statistically significant compared to the control group and resveratrol treated group kept in an enriched environment. A possible and logical explanation for this may be that EE alters the magnitude of improvement in spatial tasks imparted by the estrogen-like effect of resveratrol [52-55]. Studies comparing mice exposed to enriched and nonenriched environments have shown that, in certain tests of cognitive function, estrogen appeared to only improve performance in nonenriched mice $[53,54]$. In some of the tests, mice that both received estrogen and were kept in an enriched environment performed worse than those that received either estrogen or enrichment alone [54], which is similar to our findings. In our studies, resveratrol failed to further enhance the performance of the enriched mice beyond an improvement $(P>0.05)$ caused by EE and resveratrol treated mice alone even though both the enriched treated mice and resveratrol treated mice did not differ significantly in cognitive performance when compared to the control but did perform better than the control group and the combined treated groups. Other studies revealed similar findings when rats were exposed to mild handling stress, where estradiol failed to enhance the cognitive performance of rats beyond the improvement caused by the increased handling in radial arm maze trial [55]. This seems to indicate the occurrence of a masking or ceiling effect by enrichment; hence, if cognitive performance is improved by EE, resveratrol probably due to estrogen-like effect may not be able to further improve the cognitive performance of mice kept in an enriched environment.

Furthermore, a more convincing explanation for our findings may be that EE and histone deacetylases (HDACs) inhibitors such as trichostatin A and sodium butyrate have been identified as being capable of improving memory function in rodent experiments $[56,57]$. Some investigators reported that EE improves spatial memory capacity in mice through elevating histone acetylation in the hippocampus and further observed that the improvement in spatial memory was mimicked by HDAC inhibitors [58]. They further reported that HDAC inhibitors not only improve the capacity to form new memories but also restore the capacity to form memories in a mouse model of neurodegenerative disorders [58]. This leads to the postulation by some researchers that HDAC inhibitors could serve as a potential new therapeutic approach to human cognitive disorders and aging-related memory dysfunction arising from neurodegeneration [59].

Resveratrol which targets the sirtuin class of nicotinamide adenine dinucleotide- (NAD-) dependent deacetylases (class III histone deacetylases) mediates its beneficial effects on health and longevity in mammals through SIRT-1 NADHDACs dependent activity [60]. This further explains the failure to observe an improvement in cognitive function in resveratrol treated group kept in an enriched environment beyond the trend towards improvement caused by individual treatment of EE and resveratrol, respectively, in our procedures. While EE was acting through elevating histone acetylation via the activity of histone acetyl transferases (HATs) in the hippocampus as mentioned earlier, resveratrol was probably masking this effect through HDACs dependent activity thereby catalyzing the removal of acetyl groups from lysine residues through a $\mathrm{Zn}^{2+}$-dependent charge-relay system $[61,62]$. Thus, if acetylation of histones is functionally significant for consolidation of memory, then disruption of HAT activity would be predicted to interfere with memory formation [63].

\section{Conclusion}

In conclusion, $50 \mathrm{mg} / \mathrm{kg}$ of resveratrol administered as a caloric restriction mimetic and environmental enrichment have no significant effect on neurobehavioural responses in young healthy Swiss albino mice over a period of four weeks, although slight changes that were not statistically significant were observed in both cognitive function and motor coordination and motor endurance tests; hence, extending this study period a little longer could probably produce significant changes in neurobehavioural responses.

\section{Conflict of Interests}

The authors declare that there is no conflict of interests regarding the publication of this paper.

\section{Acknowledgments}

The authors thank Malam Mu'azu in the Department of Pharmacology and Therapeutics, Ahmadu Bello University, Zaria, Nigeria, for his assistance in training and handling of the animals. They appreciate Gombe State University, Nigeria, for providing financial assistance for the success of the work through Tertiary Education Trust Fund.

\section{References}

[1] T. S. Anekonda, "The benefits of caloric restriction and caloric restriction mimetics as related to the eye," Open Longevity Science, vol. 3, pp. 28-37, 2009.

[2] J. A. Baur, K. J. Pearson, N. L. Price et al., "Resveratrol improves health and survival of mice on a high-calorie diet," Nature, vol. 444, no. 7117, pp. 337-342, 2006.

[3] M. Obin, A. Pike, M. Halbleib, R. Lipman, A. Taylor, and R. Bronson, "Calorie restriction modulates age-dependent changes in the retinas of Brown Norway rats," Mechanisms of Ageing and Development, vol. 114, no. 2, pp. 133-147, 2000.

[4] S. Lin, E. Ford, M. Haigis, G. Liszt, and L. Guarente, "Calorie restriction extends yeast life span by lowering the level of NADH," Genes and Development, vol. 18, no. 1, pp. 12-16, 2004.

[5] G. Wolf, "Calorie restriction increases life span: a molecular mechanism," Nutrition Reviews, vol. 64, no. 2, pp. 89-92, 2006. 
[6] D. A. Sinclair, "Toward a unified theory of caloric restriction and longevity regulation," Mechanisms of Ageing and Development, vol. 126, no. 9, pp. 987-1002, 2005.

[7] R. Weindruch and R. L. Walford, The Retardation of Aging and Disease by Dietary Restriction, Charles C. Thomas, Springfield, Ill, USA, 1988.

[8] C.-K. Lee, R. G. Klopp, R. Weindruch, and T. A. Prolla, "Gene expression profile of aging and its retardation by caloric restriction,” Science, vol. 285, no. 5432, pp. 1390-1393, 1999.

[9] K. T. Howitz, K. J. Bitterman, H. Y. Cohen et al., "Small molecule activators of sirtuins extend Saccharomyces cerevisiae lifespan," Nature, vol. 425, no. 6954, pp. 191-196, 2003.

[10] J. H. Bauer, S. Goupil, G. B. Garber, and S. L. Helfand, "An accelerated assay for the identification of lifespan-extending interventions in Drosophila melanogaster," Proceedings of the National Academy of Sciences of the United States of America, vol. 101, no. 35, pp. 12980-12985, 2004.

[11] J. G. Wood, B. Rogina, S. Lavu et al., "Sirtuin activators mimic caloric restriction and delay ageing in metazoans," Nature, vol. 430, no. 7000, pp. 686-689, 2004.

[12] M. Viswanathan, S. K. Kim, A. Berdichevsky, and L. Guarente, "A role for SIR-2.1 regulation of ER stress response genes in determining C. elegans life span," Developmental Cell, vol. 9, no. 5, pp. 605-615, 2005.

[13] D. R. Valenzano, E. Terzibasi, T. Genade, A. Cattaneo, L. Domenici, and A. Cellerino, "Resveratrol prolongs lifespan and retards the onset of age-related markers in a short-lived vertebrate," Current Biology, vol. 16, no. 3, pp. 296-300, 2006.

[14] M. Lagouge, C. Argmann, Z. Gerhart-Hines et al., "Resveratrol improves mitochondrial function and protects against metabolic disease by activating SIRT1 and PGC-1alpha," Cell, vol. 127, no. 6, pp. 1109-1122, 2006.

[15] A. Anastasía, L. Torre, G. A. de Erausquin, and D. H. Mascó, "Enriched environment protects the nigrostriatal dopaminergic system and induces astroglial reaction in the 6-OHDA rat model of Parkinson's disease," Journal of Neurochemistry, vol. 109, no. 3, pp. 755-765, 2009.

[16] J. Nithianantharajah and A. J. Hannan, "Enriched environments, experience-dependent plasticity and disorders of the nervous system," Nature Reviews Neuroscience, vol. 7, no. 9, pp. 697-709, 2006.

[17] G. Laviola, A. J. Hannan, S. Macrì, M. Solinas, and M. Jaber, "Effects of enriched environment on animal models of neurodegenerative diseases and psychiatric disorders," Neurobiology of Disease, vol. 31, no. 2, pp. 159-168, 2008.

[18] E. Bezard, S. Dovero, D. Belin et al., "Enriched environment confers resistance to 1-methyl-4-phenyl-1,2,3,6-tetrahydropyridine and cocaine: involvement of dopamine transporter and trophic factors," Journal of Neuroscience, vol. 23, no. 35, pp. 10999-11007, 2003.

[19] C. J. Faherty, K. R. Shepherd, A. Herasimtschuk, and R. J. Smeyne, "Environmental enrichment in adulthood eliminates neuronal death in experimental Parkinsonism," Molecular Brain Research, vol. 134, no. 1, pp. 170-179, 2005.

[20] N. M. Jadavji, B. Kolb, and G. A. Metz, "Enriched environment improves motor function in intact and unilateral dopaminedepleted rats," Neuroscience, vol. 140, no. 4, pp. 1127-1138, 2006.

[21] B. R. Ickes, T. M. Pham, L. A. Sanders, D. S. Albeck, A. H. Mohammed, and A. Granholm, "Long-term environmental enrichment leads to regional increases in neurotrophin levels in rat brain," Experimental Neurology, vol. 164, no. 1, pp. 45-52, 2000 .
[22] T. L. Spires, H. E. Grote, N. K. Varshney et al., "Environmental enrichment rescues protein deficits in a mouse model of Huntington's disease, indicating a possible disease mechanism," Journal of Neuroscience, vol. 24, no. 9, pp. 2270-2276, 2004.

[23] B. Steiner, C. Winter, K. Hosman et al., "Enriched environment induces cellular plasticity in the adult substantia nigra and improves motor behavior function in the 6-OHDA rat model of Parkinson's disease," Experimental Neurology, vol. 199, no. 2, pp. 291-300, 2006.

[24] J. A. Baur, "Resveratrol, sirtuins, and the promise of a DR mimetic," Mechanisms of Ageing and Development, vol. 131, no. 4, pp. 261-269, 2010.

[25] D. Kim, M. D. Nguyen, M. M. Dobbin et al., "SIRT1 deacetylase protects against neurodegeneration in models for Alzheimer's disease and amyotrophic lateral sclerosis," The EMBO Journal, vol. 26, no. 13, pp. 3169-3179, 2007.

[26] L. Deng, Z.-N. Wu, and P.-Z. Han, "Effects of different levels of food restriction on passive-avoidance memory and the expression of synapsin I in young mice," International Journal of Neuroscience, vol. 119, no. 2, pp. 291-304, 2009.

[27] T. Hashimoto and S. Watanabe, "Chronic food restriction enhances memory in mice-analysis with matched drive levels," NeuroReport, vol. 16, no. 10, pp. 1129-1133, 2005.

[28] S. Yanai, Y. Okaichi, and H. Okaichi, "Long-term dietary restriction causes negative effects on cognitive functions in rats," Neurobiology of Aging, vol. 25, no. 3, pp. 325-332, 2004.

[29] L. L. Harburger, T. J. Lambert, and K. M. Frick, "Age-dependent effects of environmental enrichment on spatial reference memory in male mice," Behavioural Brain Research, vol. 185, no. 1, pp. 43-48, 2007.

[30] M. P. Mattson, W. Duan, J. Lee, and Z. Guo, "Suppression of brain aging and neurodegenerative disorders by dietary restriction and environmental enrichment: molecular mechanisms," Mechanisms of Ageing and Development, vol. 122, no. 7, pp. 757778, 2001.

[31] M. E., lia Juan, M. Pilar Vinardell, and J. M. Planas, “The daily oral administration of high doses of trans-resveratrol to rats for 28 days is not harmful," Journal of Nutrition, vol. 132, no. 2, pp. 257-260, 2002.

[32] J. Blanchet, F. Longpré, G. Bureau et al., "Resveratrol, a red wine polyphenol, protects dopaminergic neurons in MPTP-treated mice," Progress in Neuro-Psychopharmacology and Biological Psychiatry, vol. 32, no. 5, pp. 1243-1250, 2008.

[33] J. L. Stanley, R. J. Lincoln, T. A. Brown, L. M. McDonald, G. R. Dawson, and D. S. Reynolds, "The mouse beam walking assay offers improved sensitivity over the mouse rotarod in determining motor coordination deficits induced by benzodiazepines," Journal of Psychopharmacology, vol. 19, no. 3, pp. 221-227, 2005.

[34] M. Mohanasundari, M. S. Srinivasan, S. Sethupathy, and M. Sabesan, "Enhanced neuroprotective effect by combination of bromocriptine and Hypericum perforatum extract against MPTP-induced neurotoxicity in mice," Journal of the Neurological Sciences, vol. 249, no. 2, pp. 140-144, 2006.

[35] J. L. Tillerson, W. M. Caudle, M. E. Reverón, and G. W. Miller, "Detection of behavioral impairments correlated to neurochemical deficits in mice treated with moderate doses of 1-methyl-4-phenyl-1,2,3,6-tetrahydropyridine," Experimental Neurology, vol. 178, no. 1, pp. 80-90, 2002.

[36] J. Itoh, T. Nabeshima, and T. Kameyama, "Utility of an elevated plus-maze for the evaluation of memory in mice: effects of nootropics, scopolamine and electroconvulsive shock," Psychopharmacology, vol. 101, no. 1, pp. 27-33, 1990. 
[37] A. Anandhan, K. Tamilselvam, D. Vijayranjah, N. Ashokkumar, S. Rajasankar, and T. Manivasagam, "Resveratrol attenuate oxidative stress and improves behaviour in 1-methyl-4-phenyl1, 2, 3, 6-tetrahydropyridine (MPTP) challenged mice," Annals of Neuroscience, vol. 17, no. 3, pp. 113-119, 2010.

[38] J. Long, H. Gao, L. Sun, J. Liu, and X. Zhao-Wilson, “Grape extract protects mitochondria from oxidative damage and improves locomotor dysfunction and extends lifespan in a drosophila parkinson's disease model," Rejuvenation Research, vol. 12, no. 5, pp. 321-331, 2009.

[39] R. Takahashi, Y. Komiya, and S. Goto, "Effect of dietary restriction on learning and memory impairment and histologic alterations of brain stem in senescence-accelerated mouse (SAM) P8 strain," Annals of the New York Academy of Sciences, vol. 1067, no. 1, pp. 388-393, 2006.

[40] W. Duan, Z. Guo, and M. P. Mattson, "Brain-derived neurotrophic factor mediates an excitoprotective effect of dietary restriction in mice," Journal of Neurochemistry, vol. 76, no. 2, pp. 619-626, 2001.

[41] A. Wu, X. Sun, and Y. Liu, "Effects of caloric restriction on cognition and behavior in developing mice," Neuroscience Letters, vol. 339, no. 2, pp. 166-168, 2003.

[42] M. C. Roberge, J. Hotte-Bernard, C. Messier, and H. Plamondon, "Food restriction attenuates ischemia-induced spatial learning and memory deficits despite extensive CA1 ischemic injury," Behavioural Brain Research, vol. 187, no. 1, pp. 123-132, 2008.

[43] V. Tucci, A. Hardy, and P. M. Nolan, "A comparison of physiological and behavioural parameters in C57BL/6J mice undergoing food or water restriction regimes," Behavioural Brain Research, vol. 173, no. 1, pp. 22-29, 2006.

[44] M. M. Story, J. S. Rand, M. Shyan-Norwalt, R. Mesch, J. M. Morton, and E. A. Flickinger, "Effect of resveratrol supplementation on the performance of dogs in an eight-arm radial maze," The Open Nutrition Journal, vol. 6, pp. 80-88, 2012.

[45] M. K. Shigenaga, T. M. Hagen, and B. N. Ames, "Oxidative damage and mitochondrial decay in aging," Proceedings of the National Academy of Sciences of the United States of America, vol. 91, no. 23, pp. 10771-10778, 1994.

[46] E. Head, J. Liu, T. M. Hagen et al., "Oxidative damage increases with age in a canine model of human brain aging," Journal of Neurochemistry, vol. 82, no. 2, pp. 375-381, 2002.

[47] P. D. Tapp, C. T. Siwak, F. Q. Gao et al., "Frontal lobe volume, function, and $\beta$-amyloid pathology in a canine model of aging," The Journal of Neuroscience, vol. 24, no. 38, pp. 8205-8213, 2004.

[48] C. T. Siwak-Tapp, E. Head, B. A. Muggenburg, N. W. Milgram, and C. W. Cotman, "Region specific neuron loss in the aged canine hippocampus is reduced by enrichment," Neurobiology of Aging, vol. 29, no. 1, pp. 39-50, 2008.

[49] K. J. Pearson, J. A. Baur, K. N. Lewis et al., "Resveratrol delays age-related deterioration and mimics transcriptional aspects of dietary restriction without extending life span," Cell Metabolism, vol. 8, no. 2, pp. 157-168, 2008.

[50] M. Sharma and Y. K. Gupta, "Chronic treatment with trans resveratrol prevents intracerebroventricular streptozotocin induced cognitive impairment and oxidative stress in rats," Life Sciences, vol. 71, no. 21, pp. 2489-2498, 2002.

[51] Ü. Sönmez, A. Sönmez, G. Erbil, I. Tekmen, and B. Baykara, "Neuroprotective effects of resveratrol against traumatic brain injury in immature rats," Neuroscience Letters, vol. 420, no. 2, pp. 133-137, 2007.
[52] J. L. Bowers, V. V. Tyulmenkov, S. C. Jernigan, and C. M. Klinge, "Resveratrol acts as a mixed agonist/antagonist for estrogen receptors $\alpha$ and $\beta$," Endocrinology, vol. 141, no. 10, pp. 3657-3667, 2000.

[53] J. E. Gresack and K. M. Frick, "Environmental enrichment reduces the mnemonic and neural benefits of estrogen," Neuroscience, vol. 128, no. 3, pp. 459-471, 2004.

[54] J. E. Gresack, K. M. Kerr, and K. M. Frick, "Short-term environmental enrichment decreases the mnemonic response to estrogen in young, but not aged, female mice," Brain Research, vol. 1160, no. 1, pp. 91-101, 2007.

[55] J. Bohacek and J. M. Daniel, "Increased daily handling of ovariectomized rats enhances performance on a radial-maze task and obscures effects of estradiol replacement," Hormones and Behavior, vol. 52, no. 2, pp. 237-243, 2007.

[56] J. M. Levenson, K. J. O’Riordan, K. D. Brown, M. A. Trinh, D. L. Molfese, and J. D. Sweatt, "Regulation of histone acetylation during memory formation in the hippocampus," The Journal of Biological Chemistry, vol. 279, no. 39, pp. 40545-40559, 2004.

[57] W. B. Chwang, J. S. Arthur, A. Schumacher, and J. D. Sweatt, "The nuclear kinase mitogen- and stress-activated protein kinase 1 regulates hippocampal chromatin remodeling in memory formation," The Journal of Neuroscience, vol. 27, no. 46, pp. 12732-12742, 2007.

[58] A. Fischer, F. Sananbenesi, X. Wang, M. Dobbin, and L. Tsai, "Recovery of learning and memory is associated with chromatin remodelling," Nature, vol. 447, no. 7141, pp. 178-182, 2007.

[59] T. Abel and R. S. Zukin, "Epigenetic targets of HDAC inhibition in neurodegenerative and psychiatric disorders," Current Opinion in Pharmacology, vol. 8, no. 1, pp. 57-64, 2008.

[60] L. Guarente and F. Picard, "Calorie restriction-the SIR2 connection," Cell, vol. 120, no. 4, pp. 473-482, 2005.

[61] M. S. Finnin, J. R. Donigian, A. Cohen et al., "Structures of a histone deacetylase homologue bound to the TSA and SAHA inhibitors," Nature, vol. 401, no. 6749, pp. 188-193, 1999.

[62] J. J. Buggy, M. L. Sideris, P. Mak, D. D. Lorimer, B. McIntosh, and J. M. Clark, "Cloning and characterization of a novel human histone deacetylase, HDAC8," Biochemical Journal, vol. 350, no. 1, pp. 199-205, 2000.

[63] J. D. Sweatt, "Experience-dependent epigenetic modifications in the central nervous system," Biological Psychiatry, vol. 65, no. 3, pp. 191-197, 2009. 

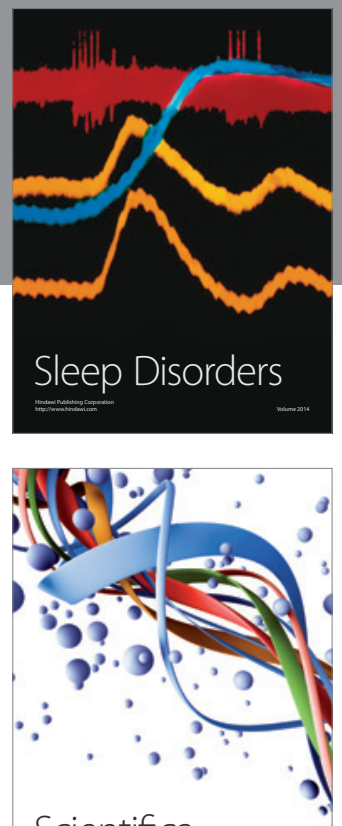

Scientifica
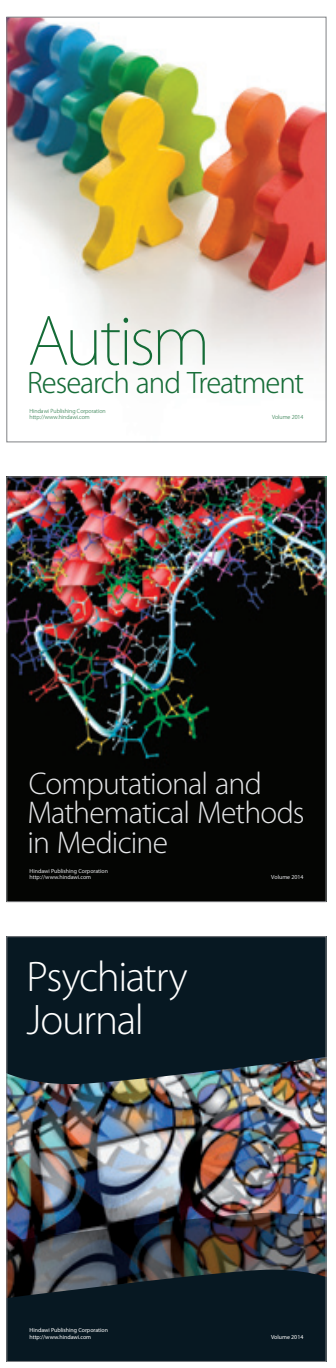
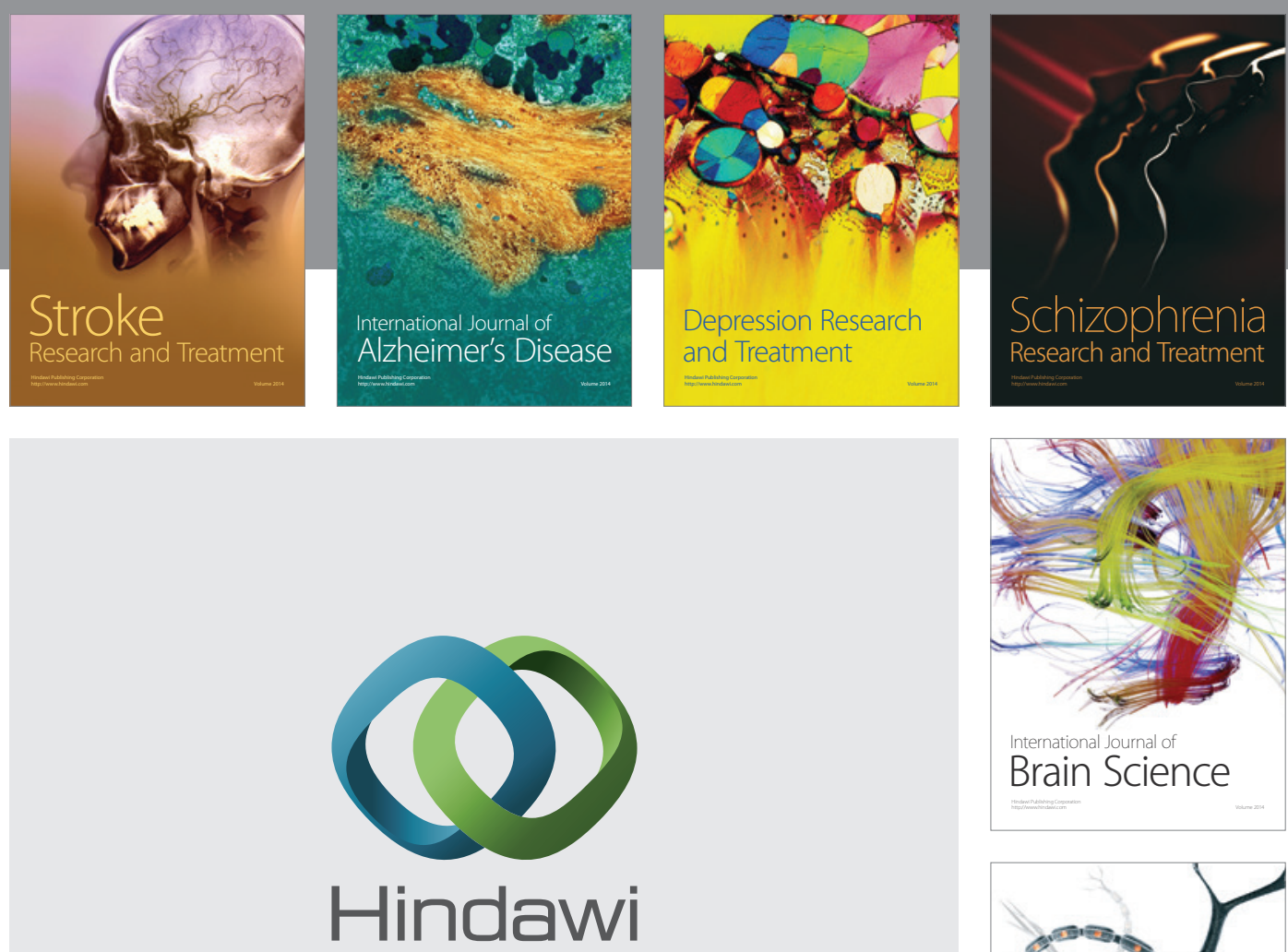

Submit your manuscripts at

http://www.hindawi.com
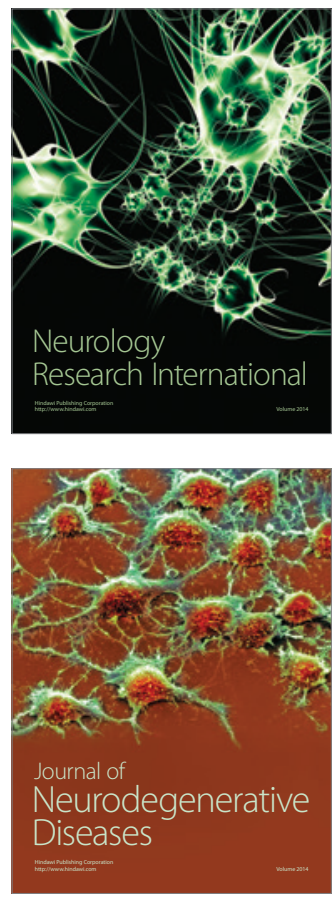

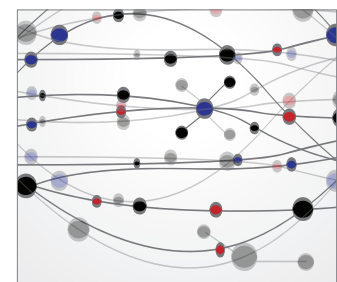

The Scientific World Journal
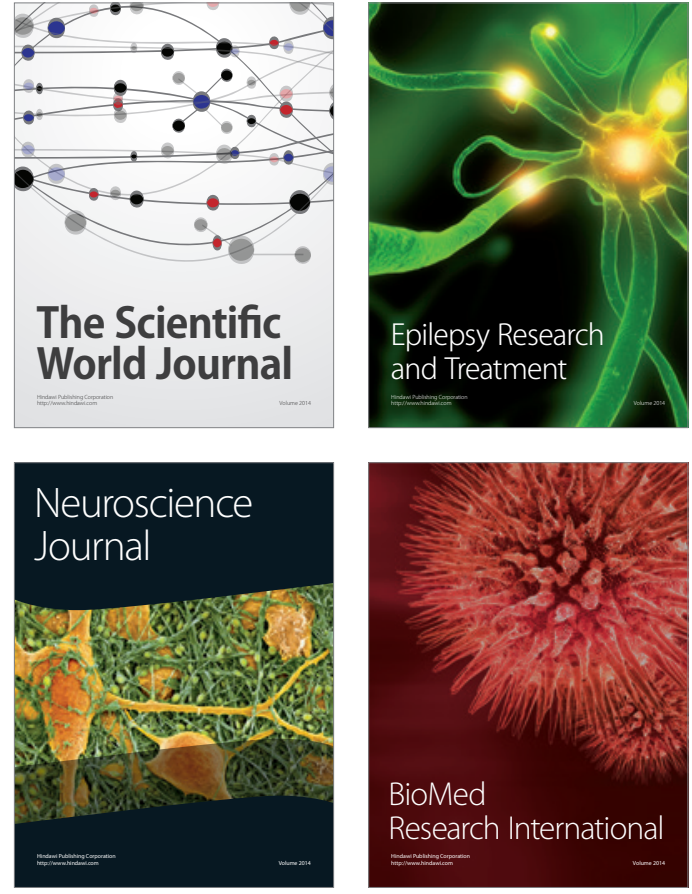

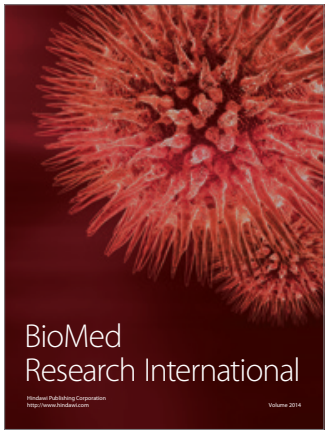

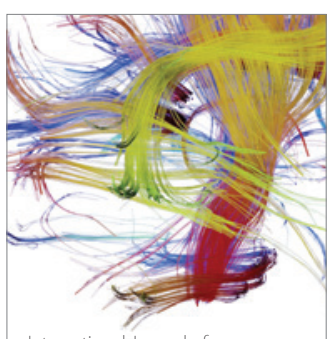

Brain Science

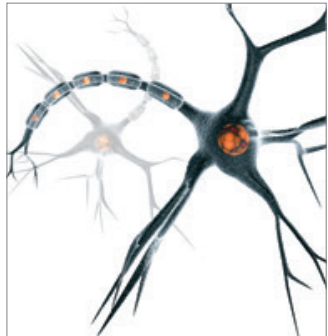

Neural Plasticity
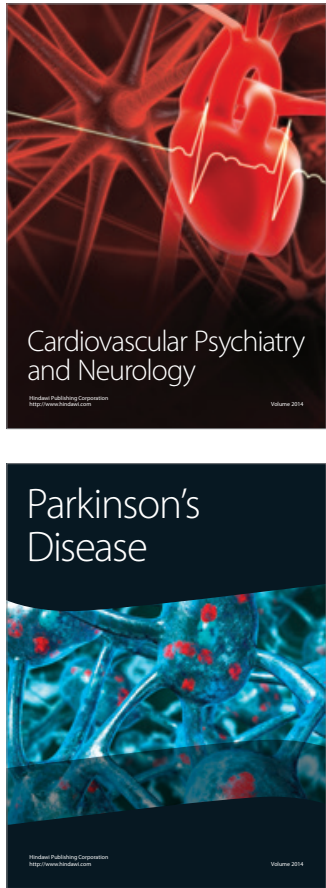